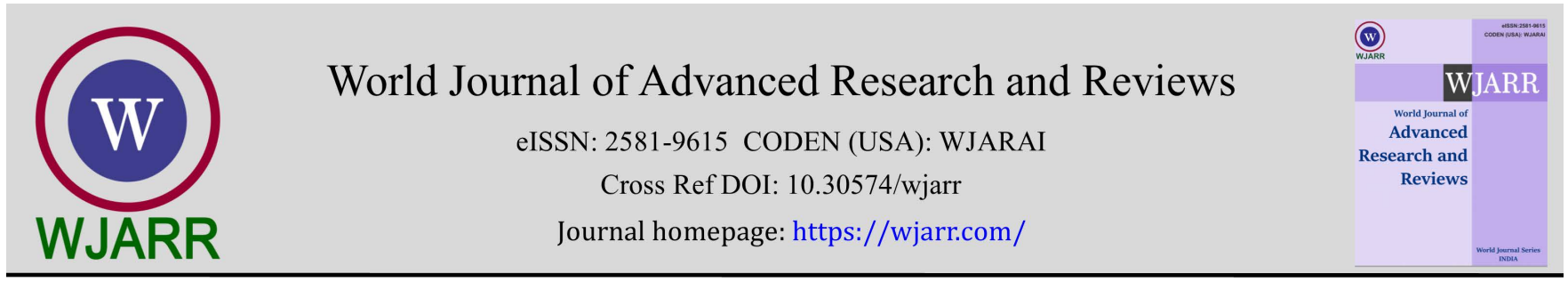

(REVIEW ARTICLE)

\title{
Electrospinning-modified $\mathrm{Pt} / \mathrm{CeO} 2$ nano bandage as a promising therapy to reduce secondary brain injury
}

\author{
Alfiani Zukhruful Fitri Rifa'i 1, Rizqi Apsari Fairuz Kamila ${ }^{1, *}$ and Clara Alverina 1, Reny I'tishom 2,* \\ ${ }^{1}$ Medical Program, Faculty of Medicine, Universitas Airlangga, Surabaya, Indonesia. \\ 2 Department of Biomedical Science, Faculty of Medicine, Universitas Airlangga, Surabaya, Indonesia.
}

World Journal of Advanced Research and Reviews, 2022, 13(01), 298-304

Publication history: Received on 30 November 2021; revised on 09 January 2022; accepted on 11 January 2022

Article DOI: https://doi.org/10.30574/wjarr.2022.13.1.0771

\begin{abstract}
Traumatic brain injury (TBI) continues to be a major contributor to morbidity, disability, and mortality in all age groups. Initial brain damage is accompanied by acute and irreversible primary damage to the parenchyma, while subsequent secondary brain damage often progresses slowly over months to years, thus providing a window for therapeutic intervention. The most frequent case which happened is excessive oxidative stress and calcium release after brain injury. Although some traditional antioxidants have been clinically approved, the efficacy is far from satisfactory due to their low ROS-scavenging efficiency, instability, toxicity, or inadequate penetration of the blood-brain barrier. Moreover, the combination of Nanozyme based-bandage with $\mathrm{Pt} / \mathrm{CeO} 2$ atom catalysis with electrospinning nanofibers $\mathrm{N}$-type voltage-gated calcium channel blocker (SNX-185) is predicted to be as promising as a potential novel to reduce secondary injury of TBI. Therefore, the duo could cut down morbidity and mortality rates because of TBI in the future, noninvasively.
\end{abstract}

Keywords: Traumatic brain injury; Secondary Injury; Nanozymes; SNX-185; Electrospinning nanofiber

\section{Introduction}

Traumatic brain injury (TBI) is structural damage and/or physiological dysfunction of the brain induced as a result of the action of an external force, causing temporary or permanent damage to the central nervous system and leading to morbidity and death [1]. The exact number of head injuries is difficult to determine due to a variety of factors. Some deaths, for example, do not reach hospitals. According to the 2018 Baseline Health Survey (Riset Kesehatan Dasar), head injuries accounted for $11.9 \%$ of the body parts affected by road traffic accidents, excluding other body parts [2].

One of the major causes of secondary injury from a TBI is an overproduction of Reactive Oxygen Nitrogen Species [3, 4]. Experimental and clinical studies have emphasized the importance of the generation of reactive oxygen species (ROS) and reactive nitrogen species (RNS) as occurring in the early post-traumatic stages of the injury process [5]. Oxidative stress (OS) leads to irreversible neuronal membrane damage, resulting in a pattern of secondary injury mechanisms ultimately leading to dysfunction and cell death [6]. Recent studies have shown evidence that neuroinflammation associated with TBI can also contribute to post-traumatic neurodegeneration $[3,7]$.

The body naturally produces endogenous antioxidants, such as superoxide dismutase and glutathione molecules, to inhibit these increases in ROS production. However, if the TBI produces more ROS than the antioxidants, lipid peroxidation, DNA, and protein damage [8]. To eliminate this excessive amount of ROS, an antioxidant is needed. One of the biggest disadvantages of traditional antioxidant bandages is that they can lose efficacy after long-term exposure at room temperature due to limited electron-donating ability [9]. Regarding this case, a nanozyme-based bandage using

\footnotetext{
* Corresponding author: Reny I'tishom

Department of Biomedical Science, Faculty of Medicine, Universitas Airlangga, Surabaya, Indonesia.

Copyright $(2022$ Author(s) retain the copyright of this article. This article is published under the terms of the Creative Commons Attribution Liscense 4.0.
} 
single-atom Pt/CeO2 with persistent catalytic activity is studied to find an alternate non-invasive treatment of neurotrauma in brain injury.

\section{Material and methods}

The search strategy for this literature review was compiled based on journal sources through search engines such as NCBI-PubMed, Research Gate, and Google Scholar online instruments. Keywords used are traumatic brain injury, Secondary Injury, Nanozymes, SNX-185, Electrospinning nanofiber with filters used are 10 years from publication date. Based on the search results, a journal containing 40 articles was selected, which is appropriate and reliable for our discussion. The level of evidence for this literature review is Level 2A based on classification under permission from the Oxford Center for Evidence-Based Medicine.

\section{Results and discussion}

\subsection{Reactive Oxygen Nitrogen Species (RONS) Timely Removal is Essential Due to Irreversible Brain Damage}

An estimated 5.3 million traumatic brain injury (TBI) survivors are currently suffering long-term or life-long neurological deficits [11]. However, currently, there are no effective neuroprotective treatments [12] because their effectiveness may be limited. In TBI, primary injury occurs at the time of trauma and the direct result is the deformation of the brain tissue and disruption of normal brain function. Secondary injury is extensive and lasting damage such as neuroinflammation with excessive oxidative stress which is believed as the main cause of neuron loss [13]. Reactive oxygen species (ROS) and reactive nitrogen species (RNS) with unpaired electrons [I4].

Mitochondrial ROS production contributes to innate immune activation after cellular damage and stress [14]. Toxicity of ROS to biomolecules causes activation of microglia and astrocytes. As a result, inflammatory and neurotoxic factors are released and increased oxidative stress, hence causing a chronic neuroinflammatory response [13].

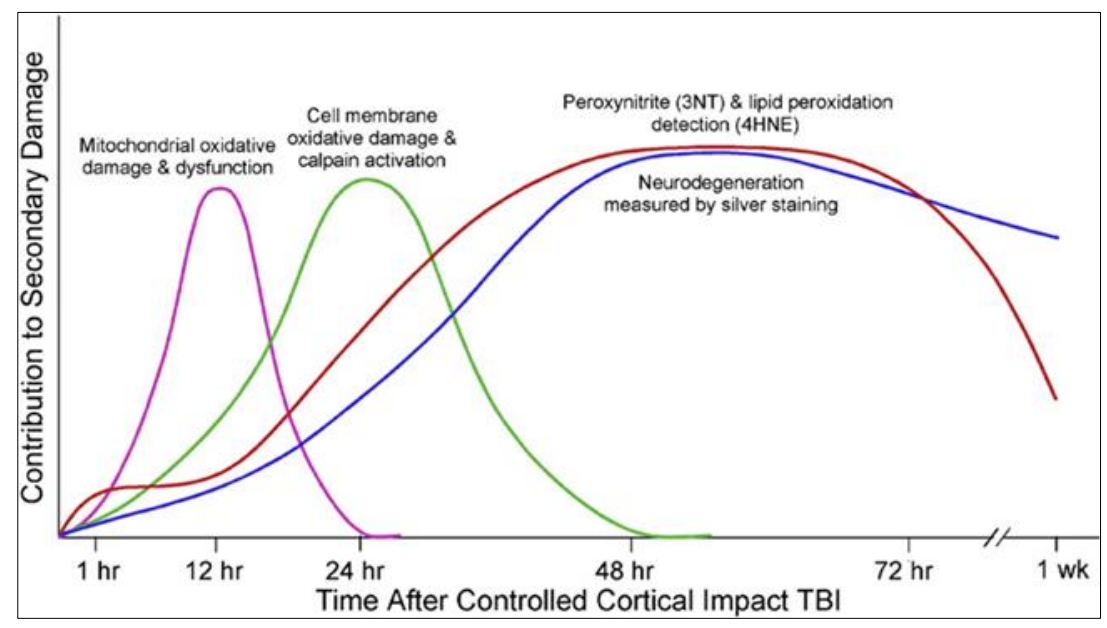

Figure 1 Time course of measured secondary injury events in the mouse CCI model of TBI [5]

Post-mortem human studies have shown that microglia activation may occur up to 17 years after TBI. This suggests that TBI induces a chronic inflammatory response, particularly in the subcortical region [15]. Therefore, it is essential to protect neurons by timely removal of harmful ROS.

\subsection{Traditional Antioxidant Has Limitation and Drawbacks for Tackling Oxidative Stress Damage in Traumatic Brain Injury}

Cell therapy has been shown to reduce neuroinflammation and improve functional recovery after TBI16. Studies of several antioxidants have shown efficacy in reducing secondary brain damage, a major focus of current TBI research $[17,18,19]$. When the redox potential is out of balance, it induces oxidative stress, which damages many macromolecules (DNA, proteins, lipids, etc.), leading to inflammation and many neurological diseases [4].

Traditional ROS regulators, such as some natural enzymes and assemblies based on them, have not been well a plied in brain diseases due to their low ROS-scavenging efficiency, instability, toxicity, or inadequate penetration of the blood- 
brain barrier [20]. For example, traditional intravenous administration of organic enzymes has shown great potential in brain diseases $[21,22]$ but toxicity remains a serious problem in clinical translation [23].

Therefore, nanotechnology can maximize the therapeutic effect while minimizing side effects [24]. Nanozyme, a stable inorganic nanomaterial with intrinsic mimetic enzyme activity, has an effective ability to reduce oxidative stress in the central nervous system. Compared with conventional antioxidants, these nanozymes have the advantages of stable structure, regulated activity, and diverse functions [20].

\subsubsection{RONS Scavenging Activity in Enzyme-mimetic Properties of single-atom Pt/CeO2 in Elimination of Excessive Oxidative Stress}

As described above, nanozymes can effectively remove ROS accumulated in the body because they have enzymemimicking activity that mainly includes SOD-like (superoxide dismutase) or catalase-like activity [20]. After TBI, a set of oxidative stress (RONS) markers are produced in the brain, while antioxidant defense enzymes are reduced (GSH, GSH/GSSG ratio, GPx, POD, SOD, CAT) [25]. Both CeO2 and Pt have SOD-like and catalase activity [26, 27]. That is, it has been reported that nanozymes have multiple antioxidant enzyme-like activities under the same conditions and exhibit an effective neuroprotective effect [20].

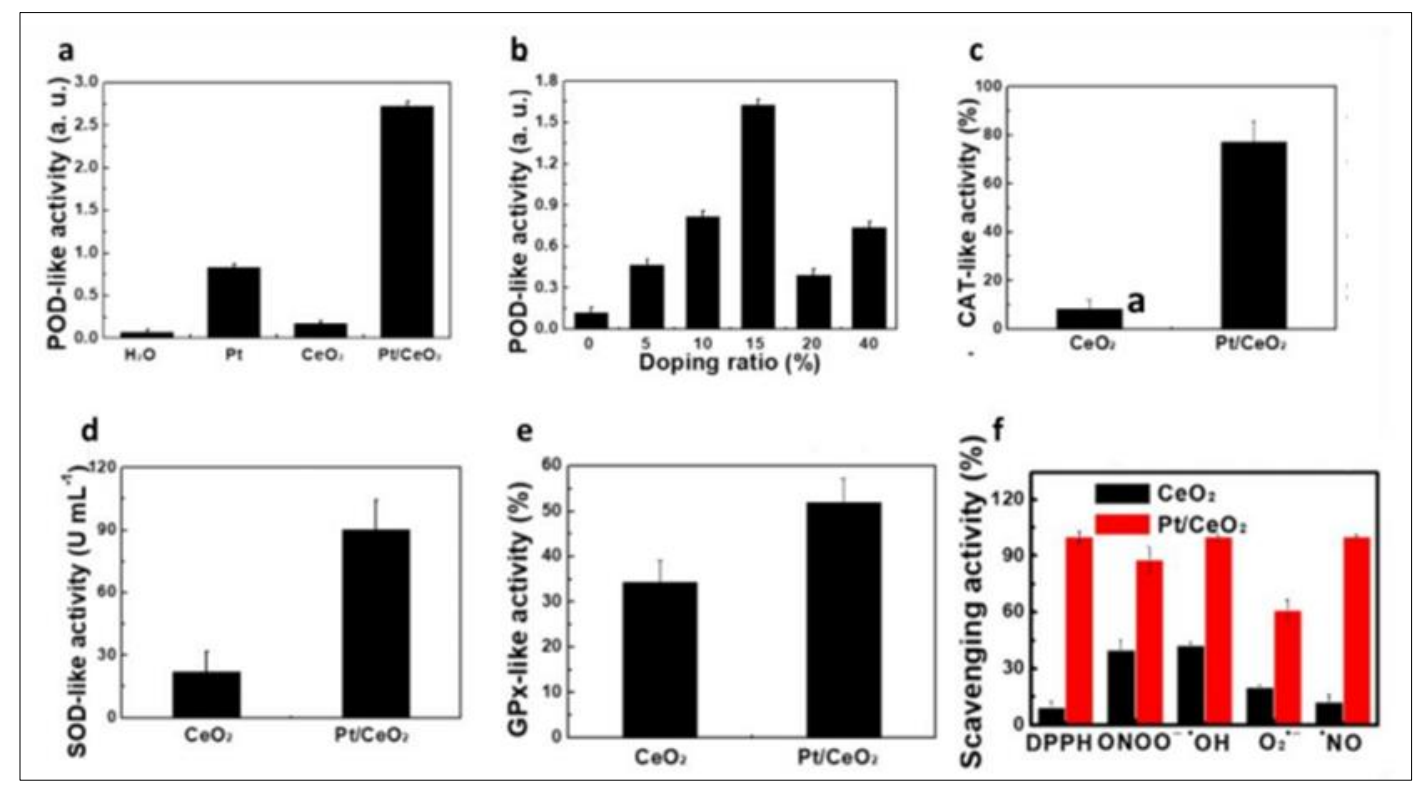

Figure 2 (a) Peroxidase (POD-like) activity after 48 h; (b) The optimal doping ratio of Pt/CeO2 is 15\% in POD, (c) CAT-like activity of Pt/CeO2 showed a 9.2times higher; (d) SOD-like activity, of Pt/CeO2 are 4 times higher; (e) GPxlike activity, (f) Pt/CeO2 catalysis endows multi-antioxidant scavenging activities [23]

The aforementioned preliminary in vitro results are for non-invasive TBI treatment with Pt/C catalysts and single-atom bands [28, 29] Pt/CeO2 showing significant catalytic activity compared to ultra-small Pt clusters [30].

\subsection{Nanozyme-Based Bandage with Single-Atom Catalysis and Nanofibers Calcium Channel Blocker with for Traumatic Brain Injury}

Nanozymes with catalytic activity represent a potential solution for the treatment of diseases related to the central nervous system and ROS [31,32]. Catalytic CeO2 and redox metal oxides captured ROS and showed protective effects against brain damage $[33,34]$.

The dressing procedure uses activated hydrophilic polyacrylonitrile fibers. They were then immersed in a special nanozyme solution for 30 minutes. After drying in vacuum and annealing for 1 hour, nanozyme-filled fibers were attached to medical tape for further application to the wound [23].

Moreover, this nanozyme-based dressing can protect neurons by eliminating ROS and reducing inflammation in neurons. Compared to the existing nanozyme intravenous injection, the dressing can be an alternative for the noninvasive treatment of TBI due to its rational design at the monoatomic level without side effects [23]. 


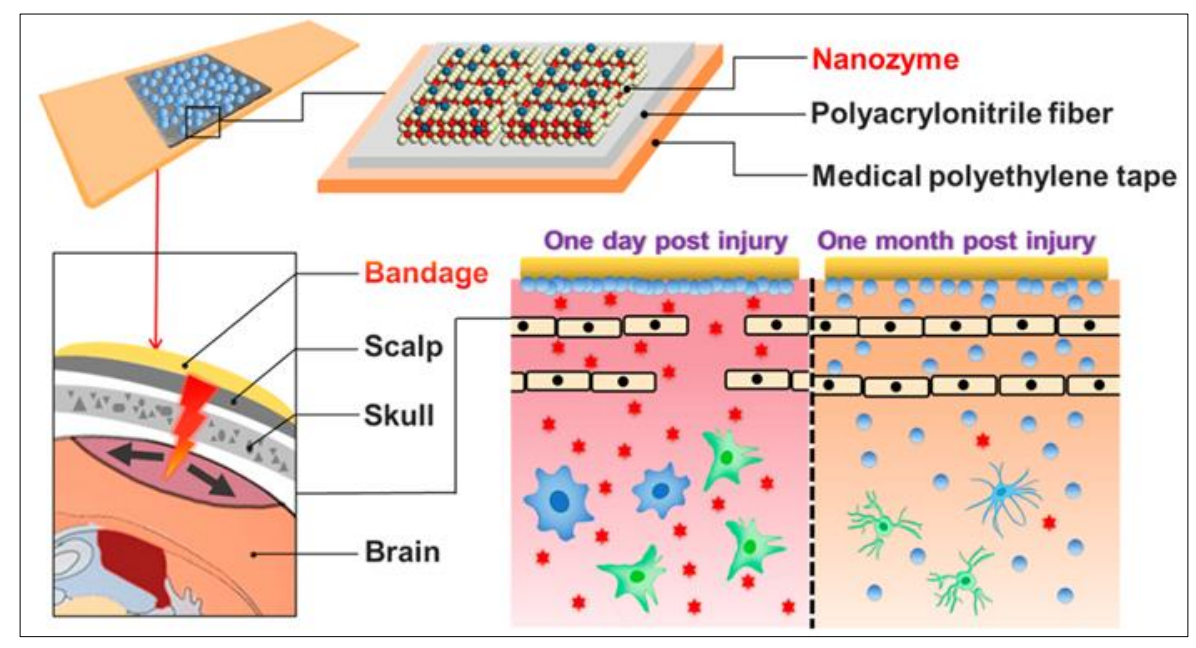

Figure 3 Concept of Using the Nanozyme-Based Bandage with Single-Atom Catalysis [23]

A nanozyme-based bandage was applied to the brain injury site of mice with TBI. Wound size and area were significantly reduced to healthy levels after dressing, whereas only partial recovery was observed in untreated mice ( 50\%) [23].

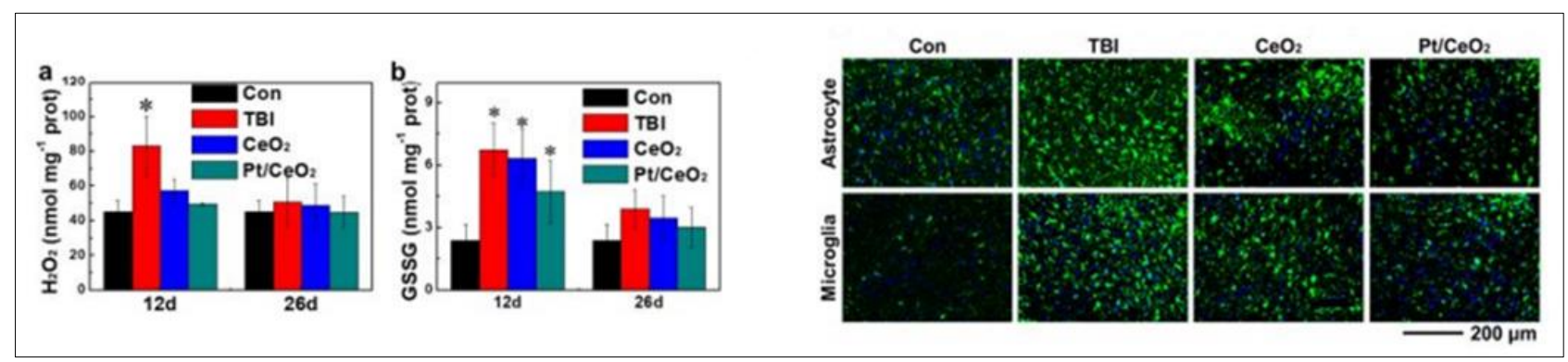

Figure 4 In vivo treatment of nanozyme bandage. (a) H2O2 generation and (b) GSSG activity (c) Immunofluorescence images for astrocyte and microglia [23]

Brain injury also mediates the expression of inflammatory cytokines and recruitment of astrocytes and microglia to the injured area, but nanozyme-based dressings reduce overall neuroinflammation by reducing inflammatory factor levels and activation of astrocytes and microglia. [23]. Both in vitro and in vivo results show that nanozyme-based dressings can reduce oxidative stress and inflammatory responses in neurons and ameliorate neurocognitive impairment. It has promising potential for the non-invasive treatment of TBI using nanozymes for single atom catalysis.

\subsection{Nanofibers N-Type Voltage-gated Calcium Channel Blocker (SNX-185) with Electrospinning Technique for Traumatic Brain Injury}

Calcium ions $(\mathrm{Ca} 2+)$ are required for the regulation of many cellular systems. Calcium dysregulation, particularly longterm increases in free calcium in the cytoplasm, can have devastating consequences for cells, leading to chronic dysfunction or death. N-type VGCC plays an important role in many developmental and pathological events in neurons and has been studied in TBI models [36, 37]. N-type channels are found in somatic cells, dendrites, and axons of neurons throughout the brain [38]. Preclinical studies conducted in vivo on an animal model of TBI have shown that selective blockade of type N VGCC leads to reduced calcium accumulation in the TBI area, preservation of mitochondrial function, and improved behavioral outcomes when administered to mice after TBI. Shows [36].

SNX-185 is an artificial model with an exceedingly selective N-kind VGCC blocker that demonstrates steed forward bioavailability and greater extended patience in mind tissue than SNX-111 [36]. These statistics propose that SNX-185 treatment, even if not on time for hours after the preliminary insult, can be powerful in preventing or lowering calciumassociated secondary accidents that arise clinically over many hours or days after injury [36]. Secondary insults including ischemia and hypoxia regularly arise after human TBI [36]. 


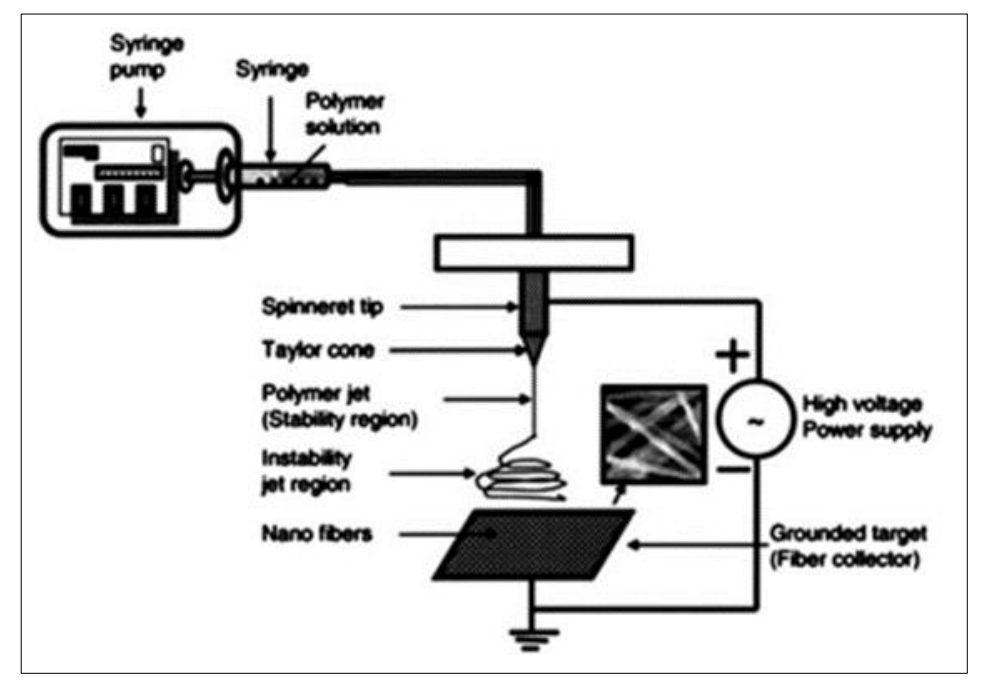

Figure 5 A schematic representation of SNX-185 Nanofibers [41]

Several strategies were said to provide nanofibers including pressurized gyration method, self-assembly, segment separation, and freeze-drying [20,39]. However, all of those strategies aren't best for wound dressing fabrication as those strategies might fail in imparting ok diffusion of oxygen and vitamins into cells ensuing in failure of cells migration, proliferation, and compensation. On the opposite situation, the electrospinning method changed into said to conquer all the formerly cited drawbacks via generating well-managed ultrafine nanofibers mimicking the extracellular matrices withinside the human body [40]. In addition, electrospinning is a cost-effective, eco-friendly, and facile method [41].

\section{Conclusion}

Nanozyme-based Bandage with Pt/CeO2 Atom Catalysis and Electrospinning Nanofibers N-Type voltage-gated Calcium Channel Blocker (SNX-185) may reduce secondary injury caused by Traumatic Brain Injury since it has a relation with RONS scavenging activity in enzyme mimetic properties by $\mathrm{Pt} / \mathrm{CeO} 2$ to eliminate the excessive oxidative stress. The combination between both elements has no specific drug interaction effect, non-invasive method, cost-effective, and eco-friendly.

\section{Compliance with ethical standards}

\section{Acknowledgments}

The author would like to thank all those who helped in writing this piece of literature.

\section{Disclosure of conflict of interest}

The authors declare that there is no conflict of interest.

\section{References}

[1] McKee Ann C, Daniel H. Daneshvar. "The neuropathology of traumatic brain injury." Handbook of clinical neurology. Elsevier. 2015; 127: 45-66.

[2] Kementerian Kesehatan, Badan Penelitian dan Pengembangan Kesehatan RI. "Hasil Utama Riset Kesehatan Dasar 2018." Departemen Kesehatan RI. 2019.

[3] Abdul-Muneer PM, Namas Chandra, James Haorah. "Interactions of oxidative stress and neurovascular inflammation in the pathogenesis of traumatic brain injury." Molecular neurobiology. 2015; 51(3): $966-979$.

[4] Russo Matthew V, Dorian B McGavern. "Inflammatory neuroprotection following traumatic brain injury." Science. 2016; 353(6301): 783-785.

[5] Bains, Mona, Edward D. Hall. "Antioxidant therapies in traumatic brain and spinal cord injury." Biochimica. et Biophysica Acta (BBA)-Molecular Basis of Disease. 2012; 1822(5): 675-684. 
[6] Bramlett, Helen M, W Dalton Dietrich. "Long-term consequences of traumatic brain injury: current status of potential mechanisms of injury and neurological outcomes." Journal of neurotrauma. 2015; 32(23): 1834 -1848.

[7] Abdul-Muneer, P M et al. "Induction of oxidative and nitrosative damage leads to cerebrovascular inflammation in an animal model of mild traumatic brain injury induced by primary blast." Free radical biology \& medicine. 2013; 60: 282-91.

[8] Salminen, Lauren E, Robert H. Paul. "Oxidative stress and genetic markers of suboptimal antioxidant defense in the aging brain: a theoretical review." Reviews in Neurosciences. 2014; 25(6): 805-819.

[9] Sood, Aditya, Mark S. Granick, and Nancy L. Tomaselli. "Wound dressings and comparative effectiveness data." Advances in wound care. 2014; 3(8): 511-529.

[10] Jeremy H. et al. "The Oxford. Levels of Evidence”. Oxford Centre for Evidence-Based Medicine. 2011.

[11] Clayton E, et al. "Brain stimulation: Neuromodulation as a potential treatment for motor recovery following traumatic brain injury." Brain research. 2016; 1640: 130- 138.

[12] Stocchetti, Nino, et al. "Neuroprotection in acute brain injury: an up-to-date review." Critical care. 2015; 19(1): 186.

[13] Hsieh, Hsi-Lung, and Chuen-Mao Yang. "Role of redox signaling in neuroinflammation and neurodegenerative diseases." BioMed research international. 2013.

[14] West, A. Phillip, Gerald S. Shadel, and Sankar Ghosh. "Mitochondria in innate immune responses." Nature Reviews Immunology. 2011; 11(6): 389.

[15] Ramlackhansingh, Anil F., et al. "Inflammation after trauma: microglial activation and traumatic brain injury." Annals of neurology. 2011; 70(3): 374-383.

[16] Crowley, Marci G, Michael G Liska, Cesar V Borlongan. "Stem cell therapy for sequestering neuroinflammation in traumatic brain injury: an update on exosome- targeting the spleen." Journal of neurosurgical sciences. 2017; 61(3): 291-302.

[17] Semple, Bridgette D. "Early preservation of mitochondrial bioenergetics supports both structural and functional recovery after neurotrauma." Experimental neurology. 2014; 261: 291-297.

[18] Zhang, Quan-Guang, et al. "Critical role of NADPH oxidase in neuronal oxidative damage and microglia activation following traumatic brain injury." PloS one. 2012; 7(4): e34504.

[19] Zhang, Qun, et al. "Resveratrol attenuates hypoxia-induced neurotoxicity through inhibiting microglial activation." International immunopharmacology. 2015; 28(1): 578-587.

[20] Xue X. The Advances of Nanozyme in Brain Disease. Nanomedicine in Brain Diseases. 2019; 149-180. Springer Nature Singapore Pte Ltd.

[21] Vernekar, Amit A., et al. "An antioxidant nanozyme that uncovers the cytoprotective potential of vanadia nanowires." Nature communications. 2014; 5: 5301.

[22] Yao, Jia, et al. "ROS scavenging Mn 304 nanozymes for in vivo anti-inflammation." Chemical science. 2018; 9(11): 2927-2933.

[23] Yan, Ruijuan, et al. "Nanozyme-Based Bandage with Single-Atom Catalysis for Brain Trauma." ACS nano. 2019; 13(10): 11552-11560.

[24] Wen, Hong, Huijeong Jung, and Xuhong Li. "Drug delivery approaches in addressing clinical pharmacologyrelated issues: opportunities and challenges." The AAPS journal. 2015; 17(6): 1327-1340.

[25] Rodriguez-Rodriguez, Ana, et al. "Oxidative stress in traumatic brain injury." Current medicinal chemistry. 2014; 21(10): 1201-1211.

[26] Fan, Jia, et al. "Direct evidence for catalase and peroxidase activities of ferritin- platinum nanoparticles." Biomaterials. 2011; 32(6): 1611-1618.

[27] Pirmohamed, Talib, et al. "Nanoceria exhibit redox state-dependent catalase mimetic activity." Chemical communications. 2010; 46(16): 2736-2738.

[28] Wang Y, Mao J, Meng X, Yu L, Deng D, Bao X. "Catalysis with two- dimensional materials confining single atoms: concept, design, and applications." Chemical reviews. 2018; 119(3): 1806-1854. 
[29] Jiao, Lei, et al. "When Nanozymes Meet Single-Atom Catalysis." Angewandte Chemie. 2019.

[30] Qiao, Botao, et al. "Single-atom catalysis of CO oxidation using Pt 1/FeO x." Nature chemistry. 2011; 3(8): 634.

[31] Zhu, Piao, Yu Chen, and Jianlin Shi. "Nanoenzyme-augmented cancer sonodynamic therapy by catalytic tumor oxygenation." Acs Nano. 2018; 12(4): 3780-3795.

[32] Xu, Bolong, et al. "A Single-Atom Nanozyme for Wound Disinfection Alications." Angewandte Chemie International Edition. 2019; 58(15): 4911-4916.

[33] Kim, Chi Kyung, et al. "Ceria nanoparticles that can protect against ischemic stroke." Angewandte Chemie International Edition. 2012; 51(44): 11039-11043.

[34] Kwon EJ, Skalak M, Lo Bu R, Bhatia SN."Neuron-targeted nanoparticle for siRNA delivery to traumatic brain injuries." ACS nano. 2016; 10(8): 7926-7933.

[35] Gurkoff, Gene G, et al. "tage-gated calcium channel blockers for the treatment of traumatic brain injury." New Therapeutics for Traumatic Brain Injury. Academic Press. 2017; 179-197.

[36] Shahlaie K, et al." Neuroprotective effects of selective N-type VGCC blockade on stretch-injury-induced calcium dynamics in cortical neurons." J. Neurotrauma. 2010; 27: 175-187.

[37] Hellmich HL, et al. Injured Fluoro-Jade-positive hippocampal neurons contain high levels of zinc after traumatic brain injury. BrainRes. 2007; 1127: 119-126.

[38] Vacher, Helene, Durga P. Mohapatra, and James S. Trimmer. "Localization and targeting of tage-dependent ion channels in mammalian central neurons." Physiological reviews. 2008; 88(4): 1407-1447.

[39] Mahalingam, S.; Edirisinghe, M. Forming of Polymer Nanofibers by a Pressurised Gyration Process. Macromol. Rapid Commun. 2013; 34: 1134-1139.

[40] Ali, Isra $\mathrm{H}$, et al. "Single-dose electrospun nanoparticles-in-nanofibers wound dressings with enhanced epithelialization, collagen deposition, and granulation properties." ACS alied materials \& interfaces. 2016; 8(23): 14453-14469.

[41] Elsherbiny IM, Ali IH. EcoFriendly Electrospin Polymeric Nanofibers Based Nanocomposites for Wound Healing and Tissue Engineering. In Eco Friendly Polymer Nanocomposites; Advanced Structured Materials; Springer: India. 2015; 75: 399-431. 